Onkologe $2021 \cdot 27: 100-107$ https://doi.org/10.1007/s00761-020-00894-4 Angenommen: 15. Dezember 2020 Online publiziert: 5. Januar 2021 (c) Der/die Autor(en) 2021

\author{
Sabine Rohrmann ${ }^{1,2} \cdot$ Silke Hermann ${ }^{3}$ \\ ${ }^{1}$ Abteilung Epidemiologie Chronischer Krankheiten, Institut für Epidemiologie, Biostatistik und \\ Prävention, Universität Zürich, Zürich, Schweiz \\ ${ }^{2}$ Krebsregister der Kantone Zürich, Zug, Schaffhausen und Schwyz, Zürich, Schweiz \\ ${ }^{3}$ Epidemiologisches Krebsregister Baden-Württemberg, Deutsches Krebsforschungszentrum, Heidelberg, \\ Deutschland
}

\title{
Onkologische Prävention - inwiefern ist die Ernährung entscheidend?
}

rückgeführt werden. Jedoch liegen die Schätzungen für die einzelnen Risikofaktoren teils deutlich auseinander [1, 7, 11, 20, 21]. Diese Unterschiede zwischen den Schätzungen der einzelnen Studien sind auf drei Dinge zurückzuführen, auf (a) die Stärke des Zusammenhangs zwischen einem Risikofaktor und einer Krebserkrankung („relatives Risiko“), (b) die Prävalenz einer Exposition/des Risikofaktors in einer Bevölkerung und (c) die relative Häufigkeit der einzelnen Krebsentitäten, die zwischen den einzelnen Ländern variiert. Die attributablen Anteile berechnen sich aus den relativen Risiken und der Prävalenz einer Exposition/eines Risikofaktors in der Bevölkerung. Kommt eine Exposition häufiger vor in einer Bevölkerung, ist der attributable Anteil auch bei gleichem Risikoschätzer größer, d. h., es werden mehr Krebsfälle durch diese Exposition erklärt. Da alle Krebsarten ein unterschiedlich hohes Präventionspotenzial haben, führen diese Unterschiede zu einem insgesamt unterschiedlichen Präventionspotenzial. Es scheint jedoch klar, dass Adipositas den stärksten Effekt auf die Krebsentstehung hat, gefolgt von Alkoholkonsum. Alle Studien zeigen für die meisten Risikofaktoren eine höhere Prävalenz bei Männern im Vergleich zu Frauen, wodurch das Präventionspotenzial bei Männern deutlich höher ist als bei Frauen [11].

Bedingt durch die Mannigfaltigkeit der einzelnen Tumorarten und deren Risikofaktoren wird eine Vielzahl wissen- schaftlicher Publikationen veröffentlicht. Dies hat zur Folge, dass der derzeitige Stand der Forschung schwer abzuschätzen ist. Um eine Übersicht der Ergebnisse aus verschiedenen Forschungsprojekten zu erhalten, evaluiert der World Cancer Research Fond (WCRF) gemeinsam mit dem American Institut for Cancer Research (AICR) kontinuierlich die publizierten Forschungsergebnisse zum Thema Ernährung und Übergewicht als Risikofaktoren und erstellt evidenzbasierte Zusammenfassungen [27]. Im Folgenden werden die wichtigsten Risikofaktoren in Bezug auf Ernährung kurz vorgestellt (• Abb. 1).

\section{Risiko erhöhende Faktoren}

Es gibt starke Evidenz, dass Übergewicht (Body-Mass-Index [BMI] 25$\left.<30 \mathrm{~kg} / \mathrm{m}^{2}\right)$ und insbesondere Adipositas $\left(\right.$ BMI $\left.\geq 30 \mathrm{~kg} / \mathrm{m}^{2}\right)$ im Erwachsenenalter ein Risikofaktor für eine Vielzahl von Krebsarten (• Abb. 1) ist [26]. Für Deutschland wird geschätzt, dass etwa $7 \%$ aller Krebserkrankungen auf zu hohes Körpergewicht zurückzuführen sind [7]; in den USA und Großbritannien sind die Zahlen ähnlich (• Tab. 1). Dabei sind die attributablen Anteile je nach Krebsentität sehr unterschiedlich; besonders stark ist der Zusammenhang für das Endometriumkarzinom (35\%) sowie für Leber- und Nierenkrebs mit je etwa $25 \%$ [7].

Der Anteil der Bevölkerung mit Adipositas steigt über die letzten Jahrzehnte 
Hier steht eine Anzeige.

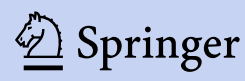


Tab. 1 Attributabler Anteil der Krebser-

krankungen infolge von Adipositas und Er-

nährungsfaktoren in verschiedenen Studien

Deutsch- USA Groß-

land [7, [17] britan-

20]

\begin{tabular}{llll} 
& 20] & & nien [11] \\
\hline Adipositas & $7,0 \%$ & $7,8 \%$ & $6,3 \%$ \\
Alkohol & $2,0 \%$ & $5,6 \%$ & $3,3 \%$
\end{tabular}

Ernährung

$\begin{array}{llll}\text { Zu viel Salz } & 0,3 \% & - & -\end{array}$

Zu viel rotes $\quad 0,4 \% \quad 0,5 \% \quad-$

Fleisch

\begin{tabular}{lll|} 
Zu viel ver- $\quad 2,0 \%$ & $0,8 \%$ & $1,5 \%$
\end{tabular}

arbeitetes

Fleisch

Zu wenig $\quad 2,0 \% \quad 1,9 \% \quad-$

Obst und

Gemüse

$\begin{array}{llll}\text { Zu wenig } & 3,0 \% & 0,9 \% & 3,3 \%\end{array}$

Ballaststoffe

Zu wenig

$0,4 \%-$

Kalzium

weltweit stetig an. Dies ist das Resultat der Tatsache, dass mehr Energie aufgenommen als verbraucht wird. Während die zu geringe körperliche Betätigung hier nicht weiter thematisiert werden soll, gehören Faktoren, die zur Aufnahme von überschüssiger Energie führen können, durchaus zu den ernährungsbedingten Risikofaktoren für Krebserkrankungen. So weisen industriell sehr stark verarbeitete Lebensmittel („ultra processed foods" [UPF]) sowohl meistens eine hohe Energiedichte als auch oft große Portionsgrößen auf. Sie tragen somit zusammen mit zuckerhaltigen Getränken zur Entwicklung von Adipositas bei [26]. Eine prospektive Studie zeigte einen stark positiven Zusammenhang zwischen dem Verzehr von UPF und dem Krebsrisiko auf: eine $10 \%$ ige Erhöhung des Anteils von UPF in der Ernährung war mit einem signifikanten Anstieg des Risikos für Gesamt- und Brustkrebs um mehr als $10 \%$ assoziiert [14]. Aufgrund der derzeitigen Forschungsergebnisse im WCRF/AICR-Bericht wird empfohlen, den Verzehr von verarbeiteten Lebensmitteln mit hohem Fett-, Stärke- oder Zuckergehalt - einschließlich „Fastfood“, vieler vorbereiteter Gerichte, Snacks, Backwaren und Desserts sowie Süßwaren (Süßigkeiten) - zu limitieren.

Der Einfluss von Adipositas auf das Krebsrisiko ist vielfältig und umfasst Ent- zündungsmechanismen und die Veränderung der Östrogen-, Androgen- und Insulinkonzentration sowie der Konzentration von Wachstumsfaktoren wie „insulin-like growth factors" [26].

Für Alkohol liegt die beste Evidenz bezüglich einer Erhöhung des Krebsrisikos vor, und so wurde Alkohol von der International Agency for Research on Cancer (IARC) bereits im Jahr 2007 als krebserregend eingestuft [6]. Basierend auf der WCRF/AICR-Evaluation ist der Zusammenhang zwischen Alkohol und dem Risiko für Tumoren des Mund- und Rachenraums, der Speiseröhre, des Magens, der Leber, des Dickdarms und Rektums sowie der Brust überzeugend oder zumindest wahrscheinlich [26]. Ein Mehrkonsum von $50 \mathrm{~g}$ Alkohol pro Tag (etwa 3 alkoholische Getränke) ist mit einer relativen Risikoerhöhung um 50 \% für Brustkrebs und um $40 \%$ für Dickdarmkrebs assoziiert [6]. Für Tumoren im oberen Atmungs- und Verdauungstrakt ist Alkoholkonsum mit einer Verdopplung bis Verdreifachung des Risikos verbunden, wobei Rauchen die Effekte des Alkoholkonsums zu verstärken scheint. Im Gegensatz dazu ist der Konsum von Alkohol möglicherweise mit einem verringerten Nierenkrebsrisiko verbunden [26].

Mons und Kollegen schätzen für Deutschland, dass etwa 2,2 \% aller Krebsfälle bei Männern $(n=8177)$ und 0,7\% bei Frauen $(n=1471)$ auf Alkoholkonsum zurückzuführen sind [20]. In der Abschätzung für Deutschland ist der attributable Anteil am höchsten für Tumoren des Mund- und Rachenraums (Männer: 34\%; Frauen: 6\%) sowie für Plattenepithelkarzinome der Speiseröhre (Männer: 30\%, Frauen: 5\%; [20]). Darüber hinaus ist der attributable Anteil von Alkohol für Dickdarm- (8,4\% bei Männern, 1,4\% bei Frauen), Leber(14,1\% bei Männern, 2,3\% bei Frauen) und Kehlkopfkrebs (18,2\% bei Männern, 3,0\% bei Frauen) sowie Brustkrebs bei Frauen (1,3\%) bedeutsam. Alle Studien kommen zum Schluss, dass Alkohol wegen des höheren Konsums bei Männern ein wichtigerer Risikofaktor ist als bei Frauen. Die Deutsche Gesellschaft für Ernährung empfiehlt, dass Männer nicht mehr als $20 \mathrm{~g}$ und Frauen nicht mehr als $10 \mathrm{~g}$ Alkohol täglich aufnehmen soll- ten [13]. $10 \mathrm{~g}$ Alkohol entspricht zum Beispiel einem Glas Bier, Wein oder Schnaps.

Alkohol kann auf verschiedenen Wegen das Krebsrisiko erhöhen. Am wichtigsten ist die Metabolisierung von Ethanol zu Acetaldehyd, das wiederum an die Desoxyribonukleinsäure (DNS) binden und damit kanzerogene Effekte auslösen kann. Daneben werden weitere krebserregende Faktoren, z. B. die Blutkonzentration von Östrogenen und die DNSMethylierung [25] durch den Konsum von Alkohol negativ beeinflusst. Zusätzlich fungiert der aufgenommene Alkohol als Lösungsmittel für Karzinogene in $\mathrm{Zi}$ garettenrauch [9].

Auch der Effekt von rotem und verarbeitetem Fleisch auf das Krebsrisiko wurde von der IARC [10] und dem WCRF/AICR [27] untersucht. Der Verzehr von verarbeitetem Fleisch wurde von der IARC als kanzerogen für den Menschen eingestuft, der Verzehr von rotem Fleisch als wahrscheinlich kanzerogen [10]. Für Deutschland wurde geschätzt, dass 2,4\% aller Krebsfälle auf den Verzehr von rotem und verarbeitetem Fleisch zurückzuführen sind, wobei sich der Konsum vor allem auf die Entstehung von Dickdarmkrebs auswirkt (12\% aller Fälle dieser Entität; [7]). Es wird davon ausgegangen, dass sich pro $50 \mathrm{~g}$ täglichem Mehrverzehr von verarbeitetem Fleisch das relative Dickdarmkrebsrisiko moderat um $18 \%$ erhöht. Um den krebserregenden Effekt von verarbeitetem Fleisch zu veranschaulichen, hat das Robert KochInstitut errechnet, dass das absolute Risiko eines 65-jährigen Mannes, in den nächsten 10 Jahren an Dickdarmkrebs $\mathrm{zu}$ erkranken, derzeit $2,4 \%$ beträgt; wird jedoch die tägliche Verzehrmenge um zusätzliche $100 \mathrm{~g}$ verarbeitetes Fleisch erhöht, steigt dieses Risiko auf $3,3 \%$ an. Für andere Tumorentitäten ist der Zusammenhang eher schwach und die Ergebnisse verschiedener Studien sind nicht eindeutig [27]. Die Deutsche Gesellschaft für Ernährung (DGE) empfiehlt eine maximale Verzehrmenge von $600 \mathrm{~g}$ Fleisch und Fleischerzeugnissen (inkl. Wurstwaren) pro Woche [13].

Die Mechanismen, die das erhöhte Dickdarmkrebsrisiko infolge von hohem 
Verzehr von rotem und verarbeitetem Fleisch bewirken, sind nicht vollständig geklärt (ausführlicher diskutiert in [24]). Unter anderem wird die direkte Aufnahme von N-Nitroso-Verbindungen (NOC; z. B. Nitrosamine) durch den Verzehr von gesalzenen und gepökelten Lebensmitteln (vor allem Fleisch, aber auch Fisch) und die NOC-Bildung im Magen-DarmTrakt als potenziell krebserregende Substanz diskutiert. Das Vorhandensein von Hämeisen scheint bei diesem Prozess eine wichtige Rolle zu spielen. Zusätzlich werden bei der Zubereitung von Fleisch, in Abhängigkeit von Garmethode, Temperatur und Dauer, heterozyklische aromatische Amine und polyzyklische aromatische Kohlenwasserstoffe gebildet, die von der IARC als kanzerogen eingestuft wurden [16]. Zusätzlich könnte eine hohe Aufnahme gesättigter Fette, die mit einem hohen Verzehr von rotem und verarbeitetem Fleisch einhergeht, für die Entstehung von Brustkrebs eine Rolle spielen.

Für Magenkrebs gilt Salz als Risikofaktor [27]. Insgesamt sind in Deutschland schätzungsweise lediglich $0,3 \%$ aller Krebsfälle, jedoch 8,7\% aller Magenkrebsfälle auf einen übermäßigen Salzkonsum zurückzuführen [7]. Die DGE gibt als Obergrenze einen Orientierungswert von bis zu $6 \mathrm{~g}$ Speisesalz/Tag (ca. $1 \mathrm{TL}$ ) an [13]. Es wird vermutet, dass das Salz die Magenschleimhaut angreifen, die endogene NOC-Bildung fördern, die Wirkung von Karzinogenen im Magen verstärken kann. Zusätzlich wird angenommen, dass Salz die Besiedlung durch Helicobacter pylori im Magen fördert [2, 12].

Derzeit wird auch die glykämische Last als Risikofaktor für das Krebsrisiko diskutiert; bisher wird ein positiver Zusammenhang mit dem Risiko für Endometriumkarzinome als wahrscheinlich angesehen [26]. Der WCRF/AICRBericht diskutiert in diesem Zusammenhang die Effekte erhöhter postprandialer Glukose- und Insulinspiegel auf die Entwicklung von Insulinresistenz, Diabetes und Adipositas, die selbst Risikofaktoren für Endometriumkarzinome darstellen [26].

Während in den Industrieländern der Verzehr von verschimmelten Nüs-

Onkologe 2021 $\cdot 27: 100-107$ https://doi.org/10.1007/s00761-020-00894-4

(c) Der/die Autor(en) 2021

\section{S. Rohrmann · S. Hermann}

\section{Onkologische Prävention - inwiefern ist die Ernährung entscheidend?}

\section{Zusammenfassung}

Hintergrund. Krebs ist eine der häufigsten chronischen Erkrankungen in Deutschland und wird zu einem beträchtlichen Teil durch veränderbare Risikofaktoren verursacht. Fragestellung. Welche Assoziationen gibt es zwischen Ernährungsfaktoren und einer Krebsentstehung?

Material und Methode. Narratives Review, das zur Beantwortung der Fragestellung vor allem systematische Übersichtsarbeiten und Metaanalysen heranzieht.

Ergebnisse. Derzeit werden alkoholische Getränke, rotes und verarbeitetes Fleisch, Salz und die glykämische Last als Risikofaktoren für zumindest einzelne Krebslokalisationen angesehen, während Obst, Gemüse,
Ballaststoffe, Kaffee und teils Milch und Milchprodukte als protektive Faktoren gelten. Im Zusammenhang mit Ernährung gilt Adipositas als wichtigster Risikofaktor, der zudem selbst durch Ernährungsfaktoren beeinflusst wird. Zusammengenommen sind diese Faktoren für etwa $18 \%$ aller Krebserkrankungen verantwortlich. Schlussfolgerungen. Auch wenn Ernährungsfaktoren nicht an das Präventionspotenzial von Rauchen heranreichen, können sie das Risiko der Tumorentstehung, vor allem im Verdauungsbereich, beträchtlich reduzieren.

Schlüsselwörter

Ernährung · Adipositas · Prävention · Krebs

\section{Oncological prevention-To what extent is nutrition important?}

\section{Abstract}

Background. Cancer is one of the most frequent chronic diseases in Germany and is to a considerable extent caused by modifiable risk factors.

Objective. Which associations exist between nutritional factors and cancer development? Material and method. Narrative review, which mainly uses systematic reviews and meta-analyses to answer the question.

Results. Currently, alcoholic beverages, red and processed meat, salt and the glycemic load are considered risk factors for at least some cancer localizations, whereas fruit, vegetables, dietary fiber, coffee and partly milk and dairy products are considered as protective factors. Obesity is considered the most important factor among the nutritional risk factors, which is itself influenced by nutritional factors. Together, these factors are responsible for approximately $18 \%$ of all cancers.

Conclusion. Even if nutritional factors do not have the preventive potential of stopping smoking, they can substantially reduce the risk of tumor development, especially in the digestive tract.

Keywords

Nutrition · Obesity · Prevention · Cancer sen und Getreideprodukten und die damit verbundene Aflatoxinaufnahme kaum eine Rolle spielt, stellt die Aufnahme dieser Substanzen in Ländern mit schlechteren Lagerbedingungen von Lebensmitteln (feucht-warmes Klima, fehlende Kühlmöglichkeiten) einen Risikofaktor für Leberkrebs dar [26].

\section{Protektive Faktoren}

Obst und Gemüse sind reich an Vitaminen und sekundären Pflanzenstoffen. Aufgrund der vielversprechenden Ergebnisse sowohl aus In-vitro-Studien als auch aus frühen Fall-Kontroll-Studien wurde diesen Lebensmitteln lange Zeit ein schützender Effekt in Bezug auf die Krebsentstehung zugesprochen. Neuere, große prospektive Studien zeigten aber, dass dieser Effekt deutlich schwächer als vermutet ist. Die Evaluation des WCRF/AICR zeigt lediglich einen wahrscheinlich vorteilhaften $\mathrm{Zu}$ sammenhang zwischen Gemüse- und Obstkonsum und dem Risiko von Tumoren im Mund- und Rachenraum [26]. Für andere Lokalisationen, zum Beispiel Dickdarm und Lunge, werden zwar auch teils protektive Effekte ge- 


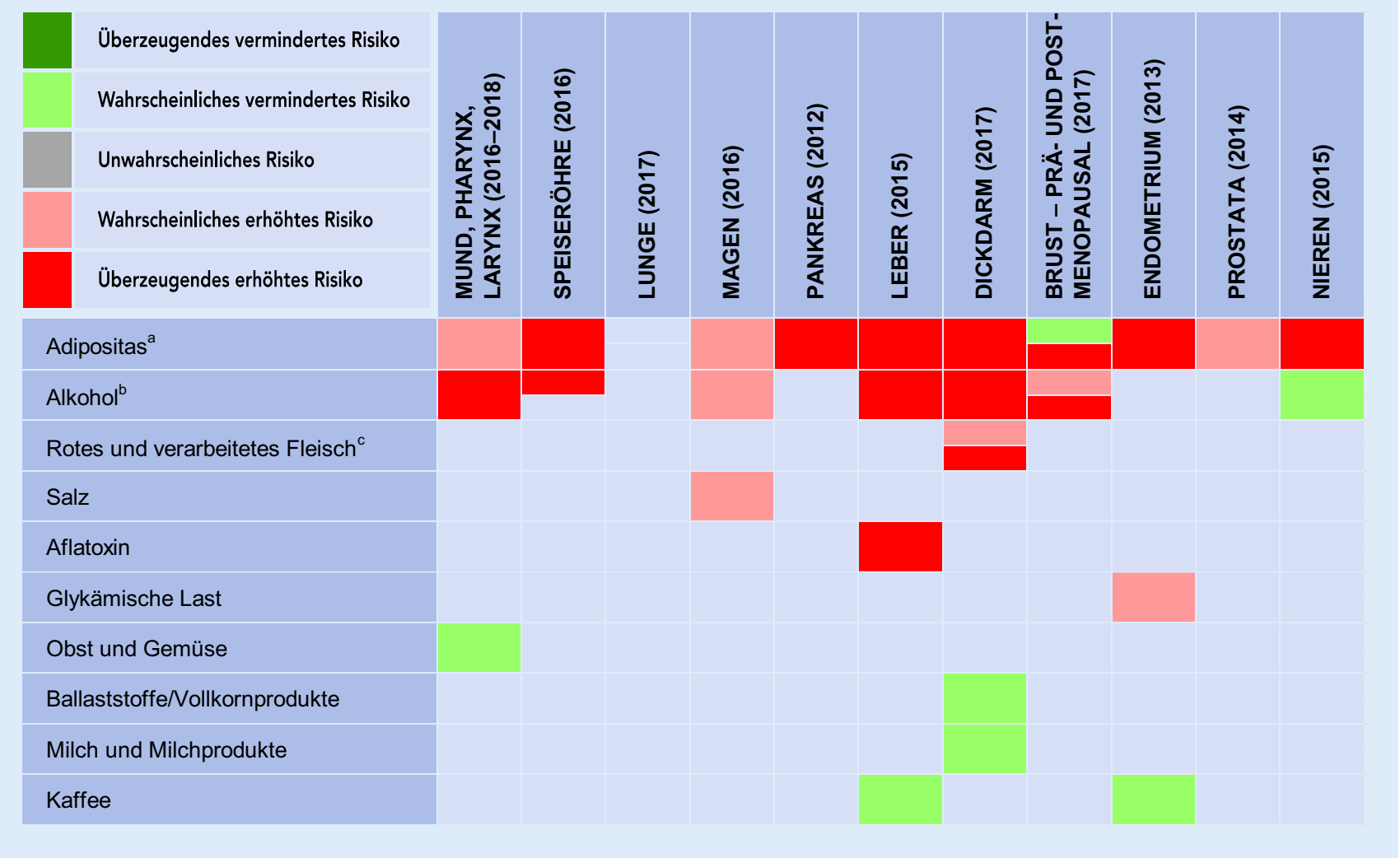

Abb. 1 \ Zusammenhänge zwischen Ernährungsfaktoren und Krebs, die vom World Cancer Research Fund/American Institute for Cancer Research als überzeugend oder wahrscheinlich eingestuft wurden. äberzeugende Evidenz nur für Adenokarzinom der Speiseröhre. Wahrscheinliche Evidenz nur für Cardiakarzinom des Magens. Überzeugende Evidenz für einen risikoerhöhenden Effekt nur für postmenopausal. Wahrscheinlich protektiver Effekt für prämenopausal. Wahrscheinlich ri-

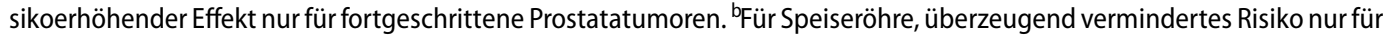
Plattenepithelkarzinom. Für Leber und Magen, nachgewiesen für Alkoholverzehrüber ca. $45 \mathrm{~g}$ pro Tag (3 alkoholische Getränke pro Tag). Für Dickdarm-Rektum, erhöhtes Risikoüberzeugend für 2+alk. Getränke pro Tag. Für Brustkrebs überzeugend nur postmenopausal; wahrscheinlich für prämenopausal. Für Nieren, nachgewiesen für Alkoholverzehr bis zu $30 \mathrm{~g}$ pro Tag (2 alkoholische Getränke pro Tag). Überzeugender Zusammenhang für verarbeitetes Fleisch; wahrscheinlicher Zusammenhang für rotes Fleisch

sehen, die aber nicht konsistent sind [26]. Eine Auswertung der EuropeanProspective-Investigation-into-Cancerand-Nutrition(EPIC)-Studie zeigte, dass ein um $200 \mathrm{~g}$ höherer Verzehr von Obst und Gemüse pro Tag das Gesamtkrebsrisiko um 3\% (95\%-Konfidenzintervall 1-4\%) senkt [8]. Für Deutschland wird das Krebspräventionspotenzial von Obst und Gemüse auf $2 \%$ geschätzt [7], ganz ähnlich der US-Einschätzung [17]. Als mögliche Erklärungen für protektive Zusammenhänge gelten die vielfältigen Effekte von Vitaminen und anderen sekundären Pflanzenstoffen. Die DGE empfiehlt eine tägliche Zufuhr von rund $400 \mathrm{~g}$ Gemüse (z. B. $200 \mathrm{~g}$ gegartes Gemüse und $200 \mathrm{~g}$ Rohkost/Salat) und etwa $250 \mathrm{~g}$ Obst [13].
Ein Teil des protektiven Effekts von Obst und Gemüse auf das Dickdarmkrebsrisiko wird wahrscheinlich über Ballaststoffe vermittelt. Der WCRF/AICR stufte den Zusammenhang zwischen der höheren Aufnahme von Ballaststoffen und einem geringeren Dickdarmkrebsrisiko als wahrscheinlich ein [27]. Laut einer Metaanalyse von 25 prospektiven Kohortenstudien senkt eine Mehraufnahme von $10 \mathrm{~g} /$ Tag das Darmkrebsrisiko relativ um $10 \%$ [3], wobei aber vor allem die Ballaststoffaufnahme aus Getreiden und Vollkornprodukten wichtig zu sein scheint. Die Schätzungen zum Präventionspotenzial gehen hier etwas auseinander: Während für Deutschland und Großbritannien eine Vermeidung von etwa $3 \%$ aller Krebsfälle als möglich gesehen wird $[7,11]$, sind es in den USA lediglich $0,9 \%$ [17]. Neben Effekten der Ballaststoffe auf die Verweildauer des Stuhls und damit möglicher Karzinogene im Darm werden die Fermentation von Ballaststoffen zu kurzkettigen Fettsäuren mit antiproliferativem Potenzial und die verminderte Bildung von sekundären Gallensäuren diskutiert [26]. Die DGE nennt eine Menge von mindestens $30 \mathrm{~g} / \mathrm{Tag}$ als Richtwert für die Zufuhr von Ballaststoffen bei Erwachsenen [13].

Kaffee gerät immer stärker in den Fokus der Forschung, und Kaffeekonsum wird nun als protektiver Faktor für einige Krebslokalisationen diskutiert. Der WCRF/AICR hält einen risikosenkenden Effekt auf das Risiko von Endometrium- und Leberkrebs für wahrscheinlich [27]. Da gerösteter Kaffee eine komplexe Mischung aus über 
Hier steht eine Anzeige.

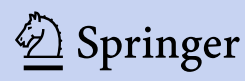


1000 bioaktiven Verbindungen ist, werden die möglichen krebshemmenden Mechanismen noch diskutiert. Einige dieser Verbindungen haben antioxidative, entzündungshemmende und/oder antifibrotische Wirkungen [22].

\section{Faktoren mit widersprüchlichen Ergebnissen}

Milch und Milchprodukte zeigen widersprüchliche Ergebnisse je nach Krebslokalisation: Während ein wahrscheinlicher protektiver Effekt eines hohen Verzehrs auf das Risiko für Dickdarmtumoren gesehen wird (pro $400 \mathrm{~g}$ Milch und Milchprodukte sinkt das Risiko relativ um 17\%; 95\%-Konfidenzintervall $12-22 \%$ [4]), geht eine hohe Kalziumaufnahme auf der anderen Seite möglicherweise mit einem erhöhten Prostatakrebsrisiko einher [5]. Als mögliche Erklärung wird diskutiert, dass hohe Kalziumkonzentrationen im Dickdarm eher die Ausdifferenzierung von Epithelzellen fördern; in der Prostata dagegen führen sie eher zur Proliferation von Zellen [19]. Bisher haben lediglich Islami et al. das Präventionspotenzial einer ausreichenden Kalziumaufnahme abgeschätzt (0,4\% aller Krebsfälle in den USA; [17]).

Zusammenfassung. Laut aktuellen Schätzungen werden etwa $18 \%$ aller Krebserkrankungen durch Adipositas, zu hohen Alkoholkonsum und verschiedene Ernährungsfaktoren verursacht. Der Konsum von verarbeitetem Fleisch und Alkohol erhöht laut Einschätzungen der IARC das Krebsrisiko. Als weitere Einflussfaktoren werden der Konsum von Obst und Gemüse sowie Kaffee (protektiv), Milchprodukte (unterschiedlich je nach Organ) und Salz (risikoerhöhend) diskutiert. Die Mechanismen sind jedoch vielfach noch unklar - so können einerseits im Lebensmittel enthaltene Substanzen wie Vitamine eine Rolle spielen, auf der anderen Seite aber auch Stoffe, die bei der Verarbeitung von Lebensmitteln entstehen. Adipositas als Folge einer zu hohen Energieaufnahme wird zudem mit einem erhöhten Risiko für multiple Krebserkrankungen assoziiert. Eine offene Frage ist, welchen Ein- fluss z.B. genetische Unterschiede oder die Darmflora auf den Zusammenhang zwischen Ernährung und Krebsrisiko haben.

\section{Fazit}

- Schätzungsweise $18 \%$ aller Krebserkrankungen werden durch Ernährung sowie durch Übergewicht und Adipositas verursacht.

- In Bezug auf die Krebsentstehung sind Adipositas, ein Übermaß an Alkohol, der erhöhte Verzehr von rotem und verarbeitetem Fleisch, Salz sowie zu geringer Konsum von Obst, Gemüse und Ballaststoffen die entscheidenden Ernährungsfaktoren.

- Den stärksten Einfluss haben Ernährungsfaktoren auf Tumoren des Verdauungstrakts.

\section{Praxistipps}

- Die Reduktion von Adipositas sollte in Bezug auf die Prävention von Krebserkrankungen erste Priorität sein. Dabei ist eine Zusammenarbeit mit professionellen Ernährungsberaterinnen und -beratern anzustreben, wobei Angebote, in denen eine Veränderung des Ernährungsverhaltens mit einer Erhöhung der körperlichen Aktivität einhergeht, zu bevorzugen sind.

- Bei einer angestrebten Veränderung der Ernährung sollten bei Personen mit einem gesunden Körpergewicht (BMI 19-25 kg/m²) folgende Punkte im Mittelpunkt stehen:

- die Energieaufnahme sollte dem Energieverbrauch entsprechen.

- den Alkoholkonsum minimieren

- den Verzehr von rotem und verarbeitetem Fleisch reduzieren

- mehr Gemüse und Obst konsumieren

\section{Korrespondenzadresse}

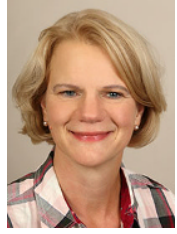

Prof. Dr. Sabine Rohrmann Abteilung Epidemiologie Chronischer Krankheiten, Institut für Epidemiologie, Biostatistik und Prävention, Universität Zürich Hirschengraben 84, 8001 Zürich, Schweiz sabine.rohrmann@uzh.ch

Funding. Open access funding provided by University of Zurich

\section{Einhaltung ethischer Richtlinien}

Interessenkonflikt. S. Rohrmann und S. Hermann geben an, dass kein Interessenkonflikt besteht.

Für diesen Beitrag wurden von den Autorinnen keine Studien an Menschen oder Tieren durchgeführt. Für die aufgeführten Studien gelten die jeweils dort angegebenen ethischen Richtlinien.

Open Access. Dieser Artikel wird unter der Creative Commons Namensnennung 4.0 International Lizenz veröffentlicht, welche die Nutzung, Vervielfältigung, Bearbeitung, Verbreitung und Wiedergabe in jeglichem Medium und Format erlaubt, sofern Sie den/die ursprünglichen Autor(en) und die Quelle ordnungsgemäß nennen, einen Link zur Creative Commons Lizenz beifügen und angeben, ob Änderungen vorgenommen wurden.

Die in diesem Artikel enthaltenen Bilder und sonstiges Drittmaterial unterliegen ebenfalls der genannten Creative Commons Lizenz, sofern sich aus der Abbildungslegende nichts anderes ergibt. Sofern das betreffende Material nicht unter der genannten Creative Commons Lizenz steht und die betreffende Handlung nicht nach gesetzlichen Vorschriften erlaubt ist, ist für die oben aufgeführten Weiterverwendungen des Materials die Einwilligung des jeweiligen Rechteinhabers einzuholen.

Weitere Details zur Lizenz entnehmen Sie bitte der Lizenzinformation auf http://creativecommons.org/ licenses/by/4.0/deed.de.

\section{Literatur}

1. American Association for Cancer Research (2020) The AACR cancer progress report 2020. AACR, Philadelphia

2. American Institute for Cancer Research, World Cancer Research Fund (2007) Food, nutrition, physical activity, and the prevention of cancer: a global perspective. AICR, Washington, DC

3. Aune D, Chan DS, Lau R et al (2011) Dietary fibre, whole grains, and risk of colorectal cancer: systematic review and dose-response metaanalysis of prospective studies. BMJ 343:d6617

4. Aune D, Lau R, Chan DS et al (2012) Dairy products and colorectal cancer risk: a systematic review and meta-analysis of cohort studies. Ann Oncol 23:37-45 
5. Aune D, Navarro Rosenblatt DA, Chan DS et al (2015) Dairy products, calcium, and prostate cancer risk: a systematic review and meta-analysis of cohort studies. Am J Clin Nutr 101:87-117

6. Baan R, StraifK,Grosse Yetal (2007)Carcinogenicity of alcoholic beverages. Lancet Oncol 8:292-293

7. Behrens G, Gredner T, Stock C et al (2018) Cancers due to excess weight, low physical activity, and unhealthy diet. Dtsch Arztebl Int 115:578-585

8. Boffetta P, Couto E, Wichmann J et al (2010) Fruit and vegetable intake and overall cancer risk in the European Prospective Investigation into Cancer and Nutrition (EPIC). J Natl Cancer Inst 102:529-537

9. Boffetta P, Hashibe M (2006) Alcohol and cancer. Lancet Oncol 7:149-156

10. Bouvard VR, Loomis D, Guyton KZ et al (2015) Carcinogenicity of consumption of red and processed meat. Lancet Oncol 16(16):1599-1600. https://doi.org/10.1016/S1470-2045(15)00444-1

11. Brown KF, Rumgay H, Dunlop C et al (2018) The fraction of cancer attributable to modifiable risk factors in England, Wales, Scotland, Northern Ireland, and the United Kingdom in 2015. Br J Cancer 118:1130-1141

12. D'Elia L, Rossi G, Ippolito Ret al (2012) Habitual salt intake and risk of gastric cancer: a meta-analysis of prospective studies. Clin Nutr 31:489-498

13. Deutsche Gesellschaft Für Ernährung (2018) Vollwertig essen und trinken nach den 10 Regeln derDGE.DGE, Bonn

14. Fiolet T, Srour B, Sellem L et al (2018) Consumption of ultra-processed foods and cancer risk: results from NutriNet-Sante prospective cohort. BMJ 360:k322

15. Giovannucci $\mathrm{E}$ (2018) A framework to understand diet, physical activity, body weight, and cancer risk. Cancer Causes Control 29:1-6

16. International Agency for Research on Cancer (1993) Heterocyclic aromatic amines. In: Some naturally occurring substances: food items and constituents, heterocyclic aromatic amines and mycotoxins. IARC, Lyon, S 165-242

17. Islami F, Goding Sauer A, Miller KD et al (2018) Proportion and number of cancer cases and deaths attributable to potentially modifiable risk factors in the United States. CA Cancer J Clin 68:31-54

18. Kerr J, Anderson C, Lippman SM (2017) Physical activity, sedentary behaviour, diet, and cancer: an update and emerging new evidence. Lancet Oncol 18:e457-e471

19. Lampe JW (2011) Dairy products and cancer. J Am Coll Nutr 30:464S-470S

20. Mons U, Gredner T, Behrens G et al (2018) Cancers due to smoking and high alcohol consumption. Dtsch Arztebl Int 115:571-577

21. Parkin DM, Boyd L, Walker LC (2011) 16. The fraction of cancer attributable to lifestyle and environmental factors in the UK in 2010. Br J Cancer 105(Suppl 2):S77-S81

22. Poole R, Kennedy OJ, Roderick P et al (2017) Coffee consumption and health: umbrella review of meta-analyses of multiple health outcomes. BMJ 359:j5024

23. Robert-Koch-Institut (2019) Krebs in Deutschland für 2015/2016. RKI, Berlin

24. Rohrmann S, Cabaset S, Linseisen J (2017)

Fleischkonsum und Krebsrisiko - darf man noch Fleisch essen? Praxis 106:871-876

25. Seitz HK, Stickel F (2007) Molecular mechanisms of alcohol-mediated carcinogenesis. Nat Rev Cancer 7:599-612

26. World Cancer Research Fund, American Institute for Cancer Research (2018) Diet, nutrition physical activity and cancer: a global perspective. Continuous update project expert report

27. World Cancer Research Fund International (2017) Continuous update project findings \& reports. Continuous update project
Hier steht eine Anzeige. Springer 